\title{
Evaluación de un test comercial de avidez de IgG: Aporte al diagnóstico de primoinfección por Toxoplasma gondii
}

\author{
Marilena Canales R., Felipe Navia G., Marisa Torres H., Mónica Concha C., \\ Ana M Guzmán D., Carlos Pérez C. y Patricia García C.
}

\section{Evaluation of an IgG avidity commercial test: Contribution to diagnosis of primary infection caused by Toxoplasma gondii}

Introduction: Toxoplasmosis $(\mathrm{T})$ is a major chronic parasitic infection in immunocompromised patients and pregnant women. It is important to discriminate between acute phase (AT) and chronic phase (CT). Diagnosis is serological in immunocompetent patients (concentration of $\operatorname{IgG}$ and $\operatorname{IgM}$ ). Objective: To evaluate the utility of an IgG avidity test (A-IgG) to identify the acute and chronic stage. Avidity is the strength of affinity between a specific immunoglobulin and the protein antigenic epitope of the infecting agent, an affinity that increases over time. Patients and Methods: We used a qualitative kit that measures the avidity of IgG, discriminating the two phases. In 35 patients with clinical diagnosis of AT and/or CT, IgG, IgM and IgG A (VIDASß) were performed. Results: Patients with AT were positive for IgM and IgG, but presented weak avidity. In the 21 cases with CT, $52 \%$ (n: 11) were IgM positive and 100\% (n: 21) had positive IgG with strong avidity. Discussion: The results confirm that the test of A-IgG may be useful in the diagnosis of AT, and has $100 \%$ concordance with reference test (qualitative IgM + quantitative IgG). The result is available within 24 hrs, and may be useful in diagnosis of AT in pregnant women.

Key words: Toxoplasmosis, serology, IgG avidity.

Palabras clave: Toxoplasmosis, serología, avidez de IgG.

\section{Introducción}

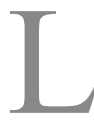

a toxoplasmosis en una infección zoonótica parasitaria cosmopolita, de amplia distribución, producida por el protozoo tisular Toxoplasma gondii. En Chile se estima una prevalencia de infección en población adulta joven de $36,9 \%{ }^{1}$. Como parte de su historia natural, esta infección cursa con dos fases: toxoplasmosis aguda (TA) y toxoplasmosis crónica (TC). La primoinfección en pacientes inmunocompetentes se presenta en $90 \%$ en forma asintomática y en $10 \%$ en forma sintomática predominando el síndrome mononucleósico ${ }^{2}$.

La primoinfección es particularmente importante durante el embarazo o cercano a él, con riesgo de transmisión transplacentaria ${ }^{3}$. En estos casos, es muy relevante el apoyo de técnicas de inmunodiagnóstico para realizar su confirmación precoz y la terapia oportuna. En pacientes inmunocomprometidos pueden observarse primoinfecciones y reactivaciones que están muy relacionadas al grado de compromiso del sistema inmune. La serología convencional en estos casos es de poca ayuda y son las técnicas de biología molecular como reacción de polimerasa en cadena para ADN las que pueden aportar al diagnóstico específico ${ }^{4-5}$.
Para realizar el diagnóstico oportuno de primoinfección durante el embarazo se requiere contar con técnicas confiables, rápidas y poco invasoras. Con este objeto algunos países realizan diagnóstico serológico de toxoplasmosis con determinación de IgM, y seroconversión de IgG (aparición de IgG en casos que no la tenían).

La detección de IgM en suero para $T$. gondii puede prolongarse por mayor tiempo de lo tradicionalmente descrito, hasta por más de un año ${ }^{6-7}$, lo que puede llevar a errores de interpretación diagnóstica. La IgG específica ha aportado al diagnóstico de la mujer embarazada especialmente a través de estudios de seroconversión. Algunos países han implementado estrategias de tamizaje poblacional pero presentan como gran limitante, el alto costo ${ }^{8-9}$.

Actualmente, se dispone de nuevas tecnologías diagnósticas basadas en el comportamiento de la inmunoglobulina $\mathrm{G}$ con el antígeno del agente infectante, determinando el grado de avidez de la IgG mediante detección por fluorescencia ELFA, Vidas ${ }^{\circledR}$, bioMerieux). Se reconoce como avidez a la fuerza de afinidad que existe entre la IgG específica y el epítope de la proteína de $T$. gondii.

De acuerdo a diferentes estudios, esta afinidad aumentaría con el tiempo, lo que permitiría discriminar si la infección es reciente ( $<4$ meses), o antigua ( $>4$ meses).

\author{
Pontificia Universidad \\ Católica de Chile, Santiago \\ Servicio de Laboratorios Clínicos \\ Laboratorio de Microbiología (MCR, \\ MCC). \\ Facultad de Medicina \\ Residente de Psiquiatría (FNG). \\ Departamento de Laboratorios \\ Clínicos (AMGD, PGC). \\ Departamento de Medicina Interna \\ (CPC).
}

Los autores declaran no tener conflictos de interés.

Fuentes de Financiamiento: Fondos del Departamento de Laboratorios Clínicos.

Recibido: 9 de mayo de 2010 Aceptado: 18 de octubre de 2010

Correspondencia a: Patricia García Cañete pgarcia@med.puc.cl 
Se reconoce que poblaciones de IgG con alta y baja avidez, existirían en forma concomitante e irían predominando según el período de la infección ${ }^{10-16}$. Entre las ventajas de la técnica de avidez de IgG se encuentra la rapidez y ser una técnica operador independiente. Es relevante destacar que en el estudio de una muestra aislada se puede contar con el resultado en menos de 24 hrs, evitando así esperar dos semanas para conocer la tendencia de las inmunoglobulinas, tiempo necesario para realizar una curva serológica.

Este estudio tiene como objetivo evaluar la utilidad de la avidez de IgG en el diagnóstico de TA en pacientes chilenos, mediante un test comercial y automatizado.

\section{Material y Método}

Se diseñó un estudio descriptivo del comportamiento del test en estudio (Toxo-IgG-Avidity ${ }^{\circledR}$ ) en comparación con los tradicionales (ELFA IgG e IgM) en muestras de pacientes con TA y TC. Los pacientes fueron atendidos en el período de dos años en la Red Salud UC y las muestras fueron procesadas en el Laboratorio de Microbiología de la Universidad Católica. Para el estudio se utilizó suero en un volumen no menor de 500 microlitros.

La avidez de la IgG se realiza en un sistema totalmente automatizado (VIDAS ${ }^{\circledR}$, bioMerieux) que utiliza urea como agente que disocia la unión IgG-antígeno. A mayor avidez, existe menor disociación de la unión antígenoanticuerpo. El resultado es un índice que relaciona el valor de fluorescencia obtenido en la muestra con agente disociante (avidez de IgG) con el valor de fluorescencia sin agente disociante (IgG), recomendándose por el fabricante los siguientes puntos de corte:

- Avidez baja: menor o igual a $200 \mathrm{UI} / \mathrm{mL}$; en estos casos se trataría de casos agudos, en el cual la infección se adquirió en los últimos cuatro meses.

- Avidez intermedia: de 200 a $300 \mathrm{UI} / \mathrm{mL}$; este rango permite descartar infección reciente, y se sugiere repetir la muestra en quince días.

- Avidez fuerte: mayor a $300 \mathrm{UI} / \mathrm{mL}$; lo que indicaría que la infección se encuentra en fase crónica, y que el paciente la adquirió hace más de cuatro meses.

Las muestras fueron estudiadas con técnicas convencionales para determinar la presencia de IgG e IgM; el test para detectar IgM es cualitativo y el test para IgG entrega resultados cuantitativos. Cuando las muestras tenían presencia de IgG pasaban a la fase de estudio de avidez de IgG. El procesamiento de esta técnica de avidez dura aproximadamente 30 minutos y puede realizarse para una o varias muestras simultaneas, con un máximo de 36. Las técnicas tradicionales utilizadas fueron ELFA IgG, IgM en el Sistema VIDAS ${ }^{\circledR}$ (bioMerieux), las que utilizan enzimo-inmunoanálisis tipo sandwich con detección fluorométrica ${ }^{17-18}$.
Se estudiaron 35 muestras serológicas, que corresponden a 35 pacientes con diagnóstico clínico de TA y TC. De éstos, 14 correspondían a TA con cuadro clínico compatible (linfoadenopatía y fiebre); 13 de ellos eran adultos inmunocompetentes, una mujer embarazada y un recién nacido con síndrome de TORCH. Como casos con TC ingresaron 21 pacientes: 2 niños y 19 adultos ( 2 mujeres embarazadas, 2 pacientes con infección por VIH/ SIDA y 15 adultos inmunocompetentes). El diagnóstico de TC se basó en el hallazgo de IgG en concentraciones positivas menores y estables en el tiempo, en pacientes que tenían antecedentes de TA sintomática previa y que actualmente estaban asintomáticos.

Análisis estadístico: Los resultados de avidez de IgG de los casos de TA se compararon con los de TC mediante un test no paramétrico de Wilcoxon. Se consideró significativo un valor $\mathrm{p}<0,05$.

\section{Resultados}

Para cada muestra se analizó la presencia de IgM, la concentración de IgG y frente a muestras con IgG positiva se determinó la avidez de IgG.

Técnicas tradicionales. En 100\% de las 35 muestras estudiadas se evidenció presencia de IgG, tanto en pacientes con TA (n: 14) como con TC (n: 21). 25 pacientes tuvieron IgM positiva, correspondiendo a $100 \%$ de las TA (14/14) y a $52 \%(11 / 21)$ de las TC (Tabla 1 y Figura 1).

En los casos de TA las concentraciones de IgG fluctuaban entre 28 y $7.184 \mathrm{UI} / \mathrm{mL}$, distribuyéndose $35,7 \%(5 / 14)$ con menos de $300 \mathrm{UI} / \mathrm{mL}, 7,1 \%(1 / 14)$ entre 300 y 700 $\mathrm{UI} / \mathrm{mL}$, y $57,1 \%$ con más de $700 \mathrm{UI} / \mathrm{mL}$ (8/14) (Tabla 2).

En los casos de TC, las concentraciones de IgG fueron menores a $300 \mathrm{UI} / \mathrm{mL}$ en $42,8 \%(9 / 21)$, entre $300-700 \mathrm{UI} /$ $\mathrm{mL}$ en $14,2 \%(3 / 21)$ y mayores de $700 \mathrm{UI} / \mathrm{mL}$ en $42,8 \%$ (Tabla 3).

Avidez de IgG. La avidez de IgG permitió discriminar, en $100 \%$ de los casos, si se trataba de TA o TC. En esta serie se observó un caso de TA con $59 \mathrm{UI} / \mathrm{mL}$ de IgG con avidez débil, y un caso de TC con IgG de 5.009 UI/ $\mathrm{mL}$ con avidez fuerte, en este último caso se encontró negativa la IgM.

De acuerdo a la distribución de los resultados según el índice de avidez, considerando el punto de corte propuesto por el fabricantes -0,3- se puede señalar que los pacientes con TA se distribuyen con una mediana de 0,1040 IC $95 \%$; 0,0383-0,0143 y los pacientes con TC presentan una mediana de 0,5500 con IC $95 \% ; 0,4874-0,5680$. Test de Wilcoxon (no paramétrico) $\mathrm{p}<0,00001$ (Figura 2). 


\begin{tabular}{|c|c|c|c|c|c|}
\hline Sexo & $\begin{array}{l}\text { Edad } \\
\text { (años) }\end{array}$ & $\begin{array}{l}\text { IgM } \\
\text { T. gondii } \\
\text { (RFV) }\end{array}$ & $\begin{array}{c}\text { IgG } \\
\text { T. gondii } \\
(\mathrm{Ul} / \mathrm{mL})\end{array}$ & Antecedentes & Diagnóstico \\
\hline $\mathrm{F}$ & 30 & 3,36 & 996 & Adenopatías sin compromiso ocular & TA \\
\hline $\mathrm{F}$ & 41 & 3,84 & 28 & Síndrome mononucleósico & TA \\
\hline M & 16 & 1,36 & 166 & Síndrome mononucleósico & TA \\
\hline $\mathrm{F}$ & 47 & 4,39 & 505 & Síndrome febril prolongado & TA \\
\hline $\mathrm{F}$ & 25 & 1,48 & 59 & Síndrome mononucleósico & TA \\
\hline M & 37 & 3,08 & 7.184 & Síndrome mononucleósico & TA \\
\hline $\mathrm{F}$ & 23 & 4,38 & 759 & & TA \\
\hline $\mathrm{F}$ & 38 & 8,43 & 237 & & TA \\
\hline M & 34 & 6,17 & 968 & Síndrome mononucleósico & TA \\
\hline $\mathrm{F}$ & 45 & 7,62 & 1.212 & Síndrome mononucleósico & TA \\
\hline $\mathrm{F}$ & 44 & 4,31 & 182 & Síndrome mononucleósico & TA \\
\hline $\mathrm{F}$ & 28 & 1,82 & 1.596 & Síndrome mononucleósico & TA \\
\hline $\mathrm{F}$ & 22 & 2,5 & 2.427 & Poliadenopatías & TA \\
\hline M & $\mathrm{RN}$ & 5,96 & 2.595 & Síndrome TORCH & TA \\
\hline $\mathrm{F}$ & 28 & 1,1 & 85 & Embarazo con antecedente de aborto & TC \\
\hline $\mathrm{F}$ & 24 & 0,69 & 510 & Antecedente de toxoplasmosis & TC \\
\hline $\mathrm{F}$ & 57 & 2,25 & 1.014 & Antecedente de toxoplasmosis & TC \\
\hline M & 21 & 1,2 & 700 & Antecedente de toxoplasmosis & TC \\
\hline $\mathrm{F}$ & 32 & 1,3 & 1.404 & Antecedente de toxoplasmosis & TC \\
\hline M & 59 & 1,54 & 4.883 & Antecedente de toxoplasmosis & TC \\
\hline $\mathrm{F}$ & 41 & 1,93 & 1.235 & Antecedente de toxoplasmosis & TC \\
\hline $\mathrm{F}$ & 30 & 0,73 & 152 & Tamizaje por embarazo & TC \\
\hline M & 20 & 1,37 & 2.916 & $\begin{array}{l}\text { Enfermedad por arañazo de gato, antece- } \\
\text { dentes de serología positiva para T. gondii }\end{array}$ & TC \\
\hline $\mathrm{F}$ & 54 & 1,41 & 85 & Bloqueo completo de rama derecha & TC \\
\hline $\mathrm{F}$ & 43 & 0,72 & 643 & Secuelas oculares & TC \\
\hline
\end{tabular}

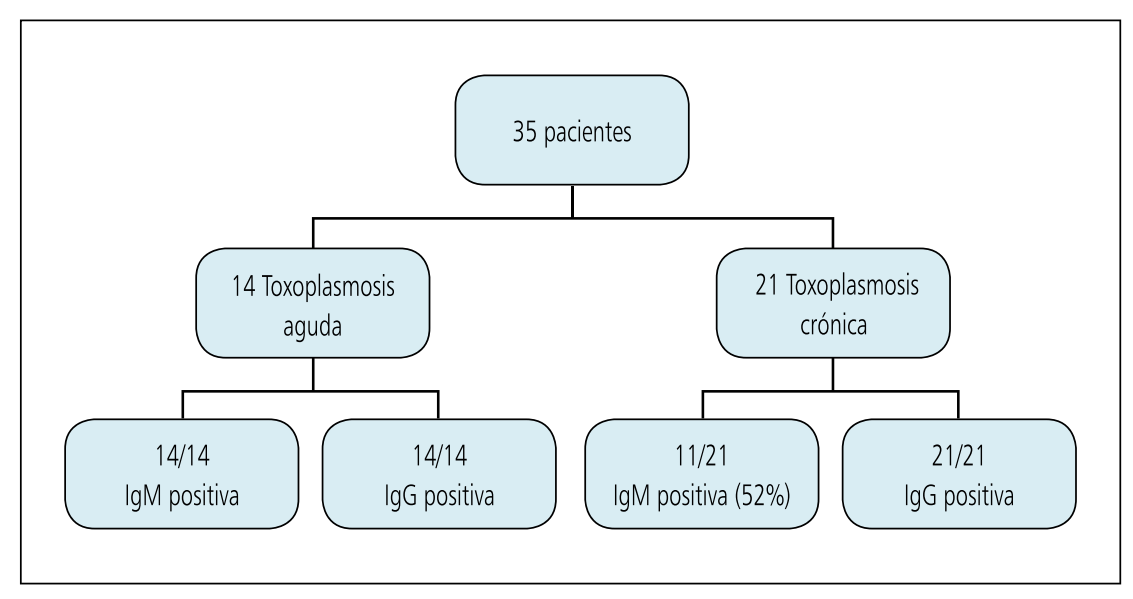

\begin{tabular}{|c|c|c|c|}
\hline \multicolumn{4}{|c|}{$\begin{array}{l}\text { Tabla 2. Distribución de la avidez de IgG } \\
\text { en } 14 \text { casos de toxoplasmosis aguda versus } \\
\text { presencia de IgM y concentración de IgG }\end{array}$} \\
\hline \multirow{2}{*}{$\begin{array}{l}\text { Resultados IgM } \\
\text { (RFV) }\end{array}$} & \multicolumn{3}{|c|}{ Avidez } \\
\hline & Fuerte & Intermedia & Débil \\
\hline IgM positiva & - & 1 & 13 \\
\hline IgM negativa & - & - & - \\
\hline \multirow{2}{*}{$\begin{array}{l}\text { Resultados IgG } \\
(\mathrm{Ul} / \mathrm{mL})\end{array}$} & \multicolumn{3}{|c|}{ Avidez } \\
\hline & Fuerte & Intermedia & Débil \\
\hline $\lg G<300$ & - & - & 5 \\
\hline $\operatorname{lgG} 300-700$ & - & - & 1 \\
\hline $\lg G>700$ & - & 1 & 7 \\
\hline
\end{tabular}

\begin{tabular}{|c|c|c|c|}
\hline \multicolumn{4}{|c|}{$\begin{array}{l}\text { Tabla 3. Distribución de la avidez de IgG } \\
\text { en los } 21 \text { casos de toxoplasmosis crónica versus } \\
\text { presencia de lgM y concentración de lgG }\end{array}$} \\
\hline \multirow{2}{*}{$\begin{array}{l}\text { Resultados IgM } \\
\text { (RFV) }\end{array}$} & \multicolumn{3}{|c|}{ Avidez } \\
\hline & Fuerte & Intermedia & Débil \\
\hline IgM positiva & 11 & - & - \\
\hline IgM negativa & 10 & - & - \\
\hline \multirow{2}{*}{$\begin{array}{l}\text { Resultados IgG } \\
(\mathrm{Ul} / \mathrm{mL})\end{array}$} & \multicolumn{3}{|c|}{ Avidez } \\
\hline & Fuerte & Intermedia & Débil \\
\hline $\lg G<300$ & 9 & - & - \\
\hline $\lg$ G 300-700 & 3 & - & - \\
\hline $\lg G>700$ & 9 & - & - \\
\hline RFV (Valor de Fluore & ia Relativ & & \\
\hline
\end{tabular}

Figura 1. Resultados de lgM e lgG anti Toxoplasma gondii en los 35 pacientes según cuadro clínico. 
Figura 2. Distribución de la avidez de $\lg G$ en pacientes con toxoplasmosis aguda versus toxoplasmosis crónica.

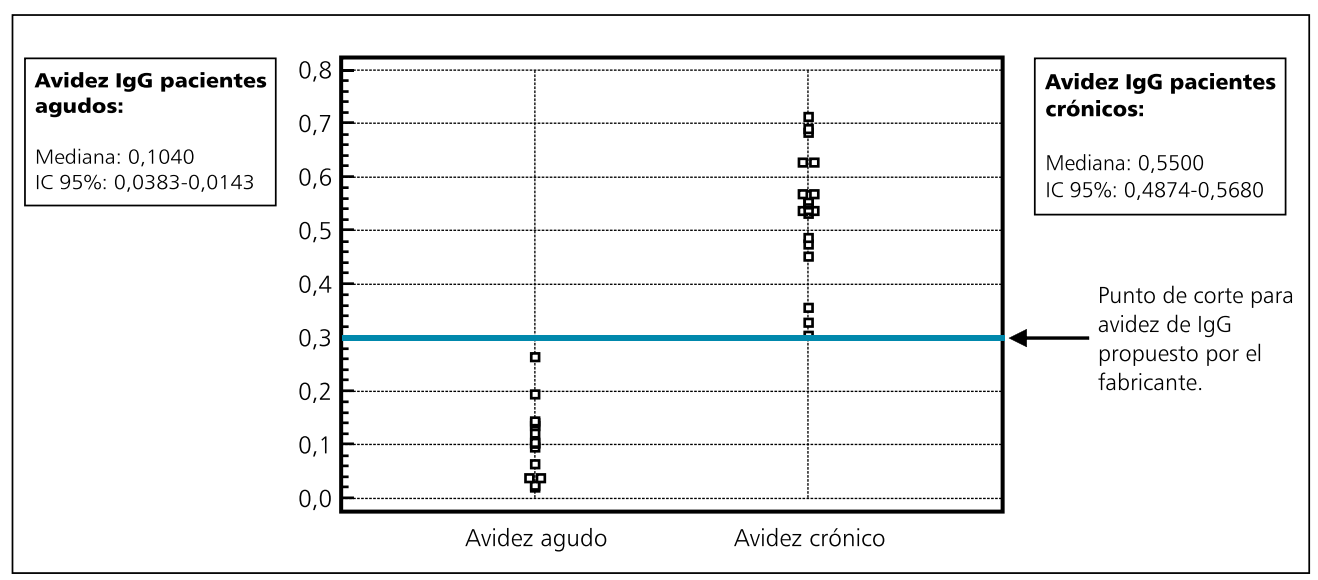

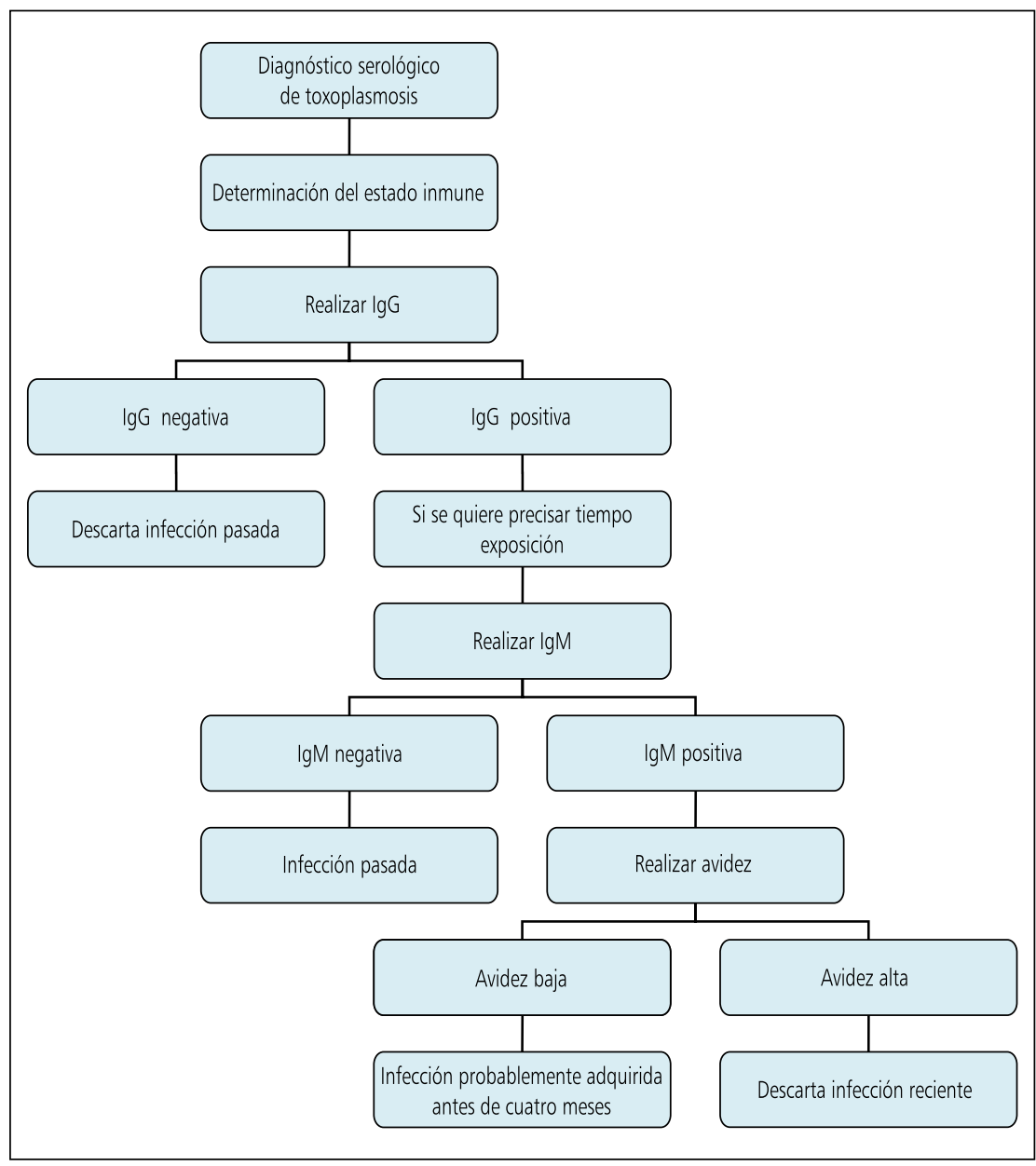

Figura 3. Algoritmo propuesto para la interpretación de los tests serológicos de toxoplasmosis en el caso de determinación del estado inmune.

\section{Discusión}

Considerando la importancia de contar con diagnósticos rápidos y oportunos de $\mathrm{TA}$, los resultados de este estudio apoyan la utilización del test de avidez de IgG como aporte al diagnóstico clínico, teniendo una excelente concordancia con los resultados de tests tradicionales (IgG + IgM), en la discriminación de fase aguda.

La avidez de IgG presenta valores significativamente más bajos en TA versus TC $(\mathrm{p}<0,00001)$. Un resultado de avidez fuerte (índice $>0,3)$ permitiría descartar una infección reciente $(<4$ meses) con bastante confiabilidad. El análisis de los resultados siempre debe ser evaluado a la luz de la clínica y de los test serológicos de apoyo, como se propone en el algoritmo (Figura 3 y 4) siguiendo las recomendaciones del CDC de Atlanta ${ }^{19}$.

Contreras y cols, el año 2000 realizó un estudio en Chile con un test de avidez no comercial con antígenos autóctonos de $T$. gondii, demostrando su utilidad en el diagnóstico confirmatorio de $\mathrm{TA}^{13}$. El contar con un test disponible comercialmente que es operador independiente favorece una implementación rápida de la técnica ofreciendo ciertos estándares de calidad en laboratorios de menor tamaño.

En Francia, la tasa de infección durante el embarazo varía desde 6,1 a 7,2 por 1.000 según la edad de la mujer embarazada y aproximadamente 300 recién nacidos nacen con toxoplasmosis congénita por año ${ }^{20}$. Considerando que en Chile la toxoplasmosis es una zoonosis prevalente, y que la población de mujeres embarazadas se encuentra en riesgo de tener primoinfección, es necesario contar con técnicas adecuadas para realizar un diagnóstico oportuno. 


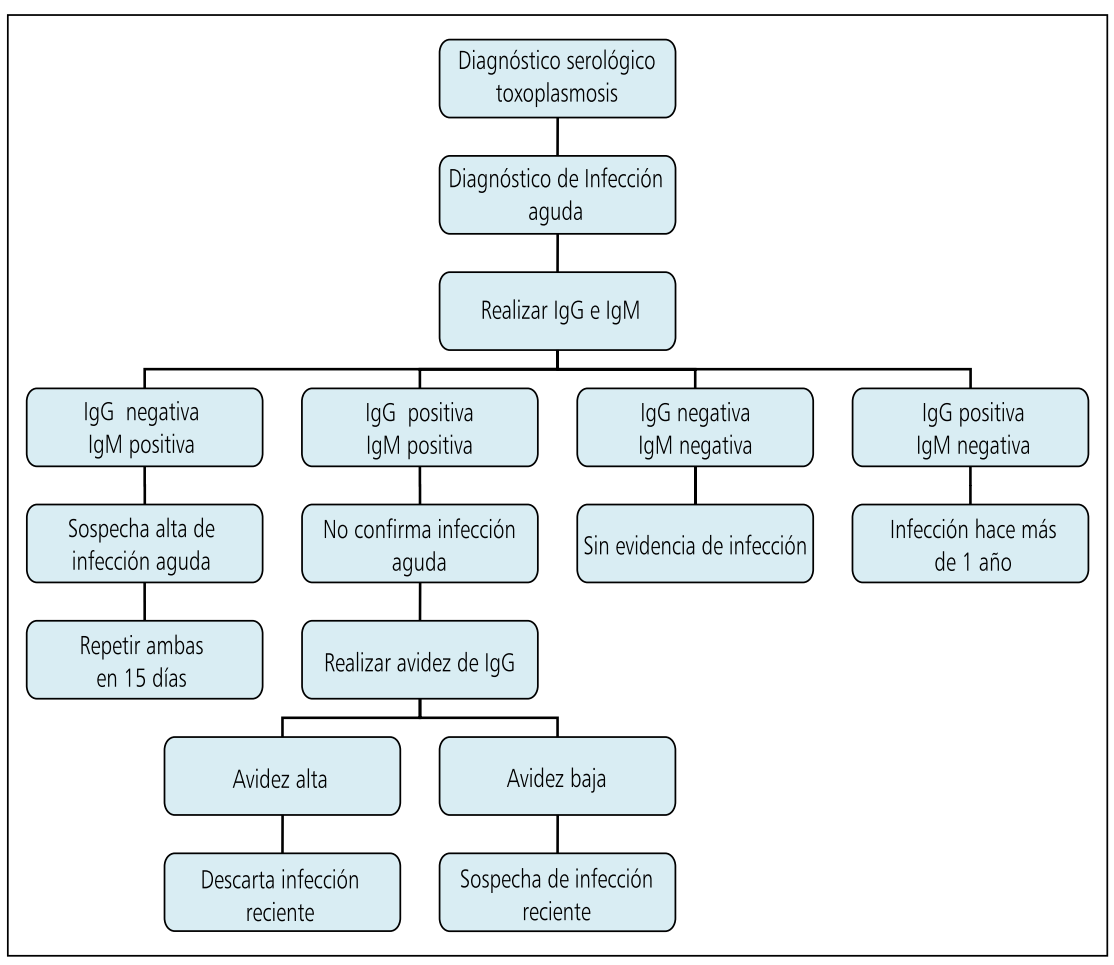

Figura 4. Algoritmo propuesto para el diagnóstico de infección aguda.

\section{Resumen}

Introducción: Toxoplasmosis (T) es una infección parasitaria crónica importante en pacientes inmunocomprometidos y mujeres embarazadas. Es relevante discriminar entre fase aguda (TA) y fase crónica (TC). Su diagnóstico es serológico en inmunocompetentes (detección de IgG e IgM). Objetivo: Evaluar la utilidad del test de avidez IgG (A-IgG) para identificar la fase aguda y o crónica. Avidez es la fuerza de afinidad entre una inmunoglobulina específica y el epítope de la proteína antigénica del agente infectante, afinidad que aumenta con el tiempo. Pacientes y Métodos: Se usó un test cualitativo que mide la avidez de $\mathrm{IgG}$, discriminando las dos fases. A 35 pacientes con diagnóstico clínico de TA y o TC, se les realizó IgG, IgM e A-IgG en Equipo VIDAS ${ }^{\circledR}$. Resultados: Los pacientes con TA fueron positivos para IgM e IgG y presentaron avidez débil. Los 21 casos con TC 52\% (n: 11) tuvieron IgM positivo y $100 \%$ (n: 21 ) tuvo IgG positiva con avidez fuerte. Discusión: Los resultados confirman que el test de A-IgG puede ser de gran utilidad en el diagnóstico de TA, concordancia: $100 \%$ con test de referencia (IgM cualitativa + IgG cuantitativa). El resultado está disponible en menos de 24 hrs, pudiendo ser útil en el diagnóstico de TA en mujeres embarazadas.

\section{Referencias}

1.- Contreras M, Schenone H, Salinas P. Seroepidemiology of human toxoplasmosis in Chile. Rev Inst Med Trop Sao Paulo 1996; 38 (6): 431-5.

2.- McCabe R E, Brooks R G, Dorfman R F, Remington J S. Clinical spectrum in 107 cases of toxoplasmic lymphadenopathy Rev Infect Dis 1987; 9 (4): 754-74.

3.- McLeod R, Kieffer F, Sautter M, Hosten T, Pelloux H. Why prevent, diagnose and treat congenital toxoplasmosis? Mem Inst Oswaldo Cruz 2009; 104 (2): 320-44.

4.- Montoya J G. Laboratory diagnosis of Toxoplasma gondii infection and toxoplasmosis. J Infect Dis 2002; 185 (Suppl 1): S73-82.

5.- Pinon J M, Dumon H, Chemla C, Franck J, Petersen E, Lebech M, et al. Strategy for diagnosis of congenital toxoplasmosis: evaluation of methods comparing mothers and newborns and standard methods for postnatal detection of immunoglobulin $\mathrm{G}, \mathrm{M}$, and A antibodies. J Clin Microbiol 2001; 39: 2267-71.

6.- Bobic B, Sibalic D, Djurkovic-Djakovic O. High levels of IgM antibodies specific for Toxoplasma gondii in pregnancy 12 years after primary toxoplasma infection. Gynecol Obstet Invest 1991; 31:182-4.

7.- Liesenfeld O, Press C, Montoya J G, Gill R,
Isaac-Renton J L, Hedman K, et al. Falsepositive results in immunoglobulin $\mathrm{M}$ (IgM) toxoplasma antibody tests and importance of confirmatory testing: the Platelia Toxo IgM test. J Clin Microbiol 1997; 35: 174-78.

8.- Desmonts G, Naot Y, Remington J S. Immunoglobulin $\mathrm{M}$-immunosorbent agglutination assay for diagnosis of infectious diseases: diagnosis of acute congenital and acquired Toxoplasma infections. J Clin Microbiol 1981; 14: 486-91.

9.- Liesenfeld O, Montoya J G, Tathineni N J, Davis M, Brown B W Jr, Cobb K L, et al. Confirmatory serologic testing for acute toxoplasmosis and rate of induced abortions among women reported to have positive Toxoplasma immunoglobulin M antibody titers. Am J Obstet Gynecol 2001; 184: 140-5.

10.- Liesenfeld O, Press C, Flanders R, Ramírez R, Remington J S. Study of Abbott Toxo IMx system for detection of immunoglobulin $\mathrm{G}$ and immunoglobulin M Toxoplasma antibodies: value of confirmatory testing for diagnosis of acute toxoplasmosis. J Clin Microbiol 1996; 34: 2526-30.

11.- Wilson M, Remington J S, Clavet C, Varney G, Press C, Ware D. Evaluation of six commercial kits for detection of human immunoglobulin $\mathrm{M}$ antibiodies to Toxoplasma gondii. J Clin
Microbiol 1997; 35: 3112-15.

12.- Hedman K, Lappalainen M, Seppala I, Makela O. Recent primary Toxoplasma infection indicated by a low avidity of specific IgG. J Infect Dis 1989; 159: 736-39.

13.- Contreras M, Sandoval L, Salinas P. Utilidad diagnóstica de ELISA IgG, IgM, IgA y ELISA avidez de IgG en toxoplasmosis reciente y crónica. Bol. Chile. Parasitol 2000; 55: 16-24.

14.- Jenum P A, Stray-Pedersen B, Gundersen A-G. Improved diagnosis of primary Toxoplasma gondii infection in early pregnancy by determination of antitoxoplasma immunoglobulin $\mathrm{G}$ activity. J Clin Microbiol 1997; 35: 1972-77.

15.- Lappalainen M, Koskela P, Koskiniemi M, Ammälä P, Hiilesmaa V, Teramo K, et al. Toxoplasmosis acquired during pregnancy: improved serodiagnosis based on avidity of IgG. J Infect Dis 1993; 167: 691-7.

16.- Liesenfeld O, Montoya J G, Kinney S, Press C, Remington J S. Effect of testing for $\mathrm{IgG}$ avidity in the diagnosis of Toxoplasma gondii infection in pregnant women: experience in a US reference laboratory. J Infect Dis 2001; 183: 1248-53.

17.- Montoya J G, Liesenfeld O, Kinney S, Press C, Remington J S. VIDAS test for avidity of Toxoplasma-specific immunoglobulin $\mathrm{G}$ for 
confirmatory testing of pregnant women. J Clin Microbiol 2002; 40: 2504-08.

18.- Pelloux H, Brun E, Vernet G. Determination of anti-Toxoplasma gondiii immunoglobulin $\mathrm{G}$ avidity: adaptation to the VIDAS system
(bioMérieux). Diagnos Microbiol Infect Dis 1998; 32 (2): 69-73.

19.- Toxoplasmosis, Antibody detection http://www. dpd.cdc.gov/dpdx/HTML/Toxoplasmosis.htm

20.- Roberts A, Hedman K, Luyasu V, Zufferey J,
Bessières M H, Blatz R M, et al. Multicenter evaluation of strategies for serodiagnosis of primary infection with Toxoplasma gondii. Eur J Clin Microbiol Infect Dis 2001; 20: 467-74. 